\title{
Topografia de Moiré como Método de Avaliação Postural: Revisão do Estado da Arte
}

\author{
MiréTopogradhy asa Mithod of Postural Evaluation Stateof theAt Peview
}

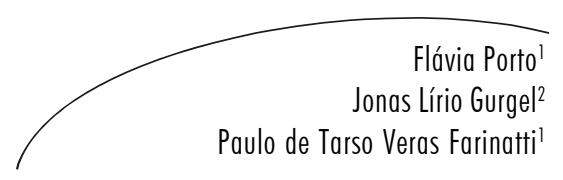

Resumo

Desde 1970, o fenômeno de moiré vem sendo empregado como método de diagnóstico clínico em análises topográficas do corpo humano. Como método de avaliação postural, a topografia de moiré (TM) auxilia na análise dos desvios posturais nos planos frontal, sagital e transverso, através de uma única fotografia (topograma) obtida do dorso do indivíduo. O objetivo deste estudo foi realizar uma revisão bibliográfica cuja abordagem incluiu as principais características do fenômeno de moiré e sua utilização como método topográfico para as análises clínicas, sobretudo aquelas relacionadas aos desvios posturais. Foi feito um levantamento bibliográfico nas principais fontes de pesquisa acadêmica internacionais e nacionais, e todo o material passou por critérios de seleção e análise preestabelecidos. Os resultados demonstraram uma evolução no método da topografia de moiré, isto é, percebeu-se uma preocupação crescente em melhorar a precisão e a acuidade do método, bem como em facilitar as interpretações dos topogramas por meio da utilização de softtwares. Em destaque, observou-se a prevalência do uso das técnicas de moiré de Sombra e de Projeção nas análises clínicas, em detrimento de outras técnicas de moiré. Somado a isso, os procedimentos metodológicos parecem não estar bem definidos na literatura. Apesar de a TM se mostrar um método de avaliação postural bastante útil na deteç̧ão de deformidades da coluna vertebral, verificou-se, por outro lado, grande carência de estudos que a tivessem aplicado em adultos ou idosos. A prevalência está em estudos de rastreamento de escolioses em crianças em idade escolar, por se tratar de um problema de saúde pública.

\footnotetext{
1 Laboratório de Atividade Física e Promoção da Saúde, Instituto de Educação Física e Desportos, Universidade do Estado do Rio de Janeiro e Programa de Pós-graduação Stricto Sensu em Educação Física, Universidade Gama Filho. Rio de Janeiro, RJ, Brasil.

2 Instituto de Educação Física. Universidade Federal Fluminense. Campus Esportivo do Gragoatá. Niterói, RJ, Brasil

Apoio: Este estudo foi subvencionado por meio de bolsa de Doutorado pelo Programa de Suporte à Pós-Graduação de Instituições de Ensino Particulares da Coordenação de Aperfeiçoamento de Pessoal de Nível Superior (PROSUP/CAPES/ Brasil).

Correspondência / Correspondence

Flávia Porto

Laboratório de Atividade Física e Promoção da Saúde, Instituto de Educação Física e Desportos.

Universidade do Estado do Rio de Janeiro (LABSAU, IEFD, UERJ)

Rua São Francisco Xavier, n 524 - sala 8.133 - bloco F, Maracanã

20550-013 Rio de Janeiro, RJ, Brasil

E-mail: flaviaporto_@terra.com.br
}

Palavras-chave: Topografia de Moiré. Diagnóstico. Postura. Escoliose. Coluna Vertebral. Revisão. 


\section{Abstract}

Since 1970, the moiré phenomenon has been employed as a method of clinical diagnosis in topographical analyses of the human body. As a postural evaluation method, the moiré topography (MT) assists in the examination of postural deviations on frontal, sagittal and transversal planes, through the analysis of a single image (topogram) of the subject's backside. This study aimed to carry out a literature review whose approach included the main characteristics of the moiré phenomenon and its use as a topographical method for clinical analyses, particularly those related to postural deviations. A literature survey of the main sources of international and national academic research was made and all the material collected from this survey then underwent a pre-established selection criteria and analysis. The results showed an evolution in the method of moiré topography, that is, an increasing concern to improve the accuracy and precision of the method, as well as to facilitate the interpretations of the topograms by means of the use of softwares. It was mainly observed that the shadow and projection moiré techniques were more used than the other techniques of moiré. In addition to this, it was apparent that the methodological procedures of moiré topography are not well defined in literature. Although MT has shown to be very useful in the detection of vertebral column deformities, it was verified that there exists a great lack of studies that have applied this technique to clinically evaluate adults and the elderly. The majority of studies involve the tracking of scoliosis in school age children, because it is a public health issue.
Key words: Moiré Topography. Diagnosis. Posture. Scoliosis. Spine. Review.

\section{INTRODUÇÃO}

A introdução do fenômeno de moiré em estudos científicos deu-se em 1874, por Raylegh, quando comparou a sobreposição de duas grades iguais. ${ }^{1}$ A utilização do moiré permite avaliar a topografia de uma superfície porque as franjas de moiré formam linhas de contorno ou curvas de nível do objeto em análise. ${ }^{2}$ Há diversas técnicas que possibilitam a geração de moiré, sendo que a técnica de moiré de sombra foi a precursora para análises da superfície do corpo humano, no caso o tronco, o que ocorreu somente na década de $1970 .^{3}$

Desde a proposta de Takasaki no emprego da topografia de moiré (TM) para a análise da superfície do corpo humano, outros autores publicaram estudos com o objetivo de explicar, aperfeiçoar e empregar o método em indivíduos de diferentes características, bem como utilizar outras possibilidades de técnicas que a TM abrange. ${ }^{2,4-12} \mathrm{O}$ próprio Takasaki, inclusive, pareceu preocupar-se em acompanhar a evolução da técnica e suas diversas possibilidades de aplicações, ${ }^{13-15}$ após sua primeira publicação, em 1970.

Ainda assim, atualmente, poucos instrumentos voltados à avaliação da superfície corpórea humana são disponibilizados no mercado - embora se reconheçam as vantagens da TM como ferramenta diagnóstica e de profilaxia de eventos clínicos, especialmente os desvios posturais da coluna. Talvez essa lacuna percebida se deva ao pouco conhecimento sobre o fenômeno físico de moiré e, consequentemente, às suas opções de aplicação na área médica.

Estruturar e organizar uma área de conhecimento facilita a produção de estudos futuros, evitando redundância e má orientação da pesquisa. Acredita-se que o presente trabalho se justifica por contribuir para a literatura científica da área e por motivar os profissionais da saúde a fazerem uso da TM em suas avaliações clínicas.

Deste modo, o objetivo deste estudo foi apresentar uma revisão bibliográfica sobre as 
principais características do fenômeno de moiré e sua utilização como método topográfico para diagnósticos clínicos, sobretudo os relativos a desvios posturais. O perfil de sujeitos frequentemente avaliados também foi avaliado. A hipótese trabalhada foi que pessoas jovens e idosas são avaliadas com a TM.

\section{MATERIAIS E MÉTODOS}

Para a constituição do corpus de análise desta revisão narrativa, a pesquisa de levantamento foi dividida em duas fases. Na primeira, foi feita uma busca de estudos nas fontes de pesquisa acadêmica Pubmed, Science Direct e Google Acadêmico. Também foi utilizada a ferramenta de Pesquisa Múltipla, disponível no website da Biblioteca Irmão José Ótão, da Pontifícia Universidade Católica do Rio Grande do Sul (PUC-RS). Esta ferramenta permite, com apenas um comando, realizar pesquisas múltiplas em diversas fontes de informação disponíveis na Biblioteca da PUC-RS. Desta forma recupera, de uma só vez, artigos científicos em texto completo disponíveis em bases de dados eletrônicas, dentre elas: Proquest, Ebsco, Biological Abstracts, Scielo e PubMed. Somado a isso, alguns trabalhos foram adquiridos por meio de contato direto com os autores, via correio eletrônico (e-mai). Já na segunda fase, foi feito um levantamento bibliográfico baseado na lista de referências de cada trabalho encontrado.

Para este estudo, o critério de busca e seleção dos artigos foi o rastreamento de material bibliográfico nas fontes supracitadas através de termos-chave, nos idiomas português e inglês, incluindo: moiré, moiré topography, shadow moiré e moiré technique. Também foram consultados livros-texto de física, com abordagem em óptica (Optics source book e Optical measurement methods in biomechanics).

Todas as obras literárias obtidas foram submetidas à técnica de scanning (Goodman, 1976 apud $\left.^{16}\right)$, que corresponde a uma leitura superficial dos artigos com o intuito de melhor selecionar os trabalhos úteis ao desenvolvimento do estudo.
Alguns artigos cujas publicações são anteriores a 1980, apesar de já não se apresentarem atuais do ponto de vista de descobertas científicas, foram importantes para a caracterização do estado da arte da TM e obtidos através do Serviço de Comutação, também disponível no sítio da Biblioteca Irmão José Ótão da PUC-RS.

No material selecionado, foi aplicada uma análise de conteúdo adaptada da proposta de Bardin ${ }^{17}$ e utilizada em outros estudos. ${ }^{18}$ Como características de análise, foram adotadas: 1) a descrição do fenômeno de moiré e a origem da palavra estrangeira; 2) as técnicas de avaliação baseadas nesse fenômeno físico, incluindo os instrumentos empregados, a calibração desses equipamentos, a análise da imagem e os cuidados na aplicação; 3) as áreas médicas de aplicação.

Este estudo abordou o tema sob o formato de tópicos, sendo eles: o princípio físico de moiré, as técnicas baseadas no fenômeno de moiré, a calibração dos equipamentos para a aplicação da técnica, a análise dos topogramas (ou seja, as imagens de moiré), as aplicações do método e os cuidados necessários para o emprego da TM.

\section{RESULTADOS E DISCUSSÃO}

A palavra moiré deriva do termo francês "molhado" ou "ondulado", para designar um tecido de seda importado da China, no qual os fios fornecem uma aparência peculiar ao pano. ${ }^{19}$, ${ }^{20}$ No Brasil, o tecido de mesma aparência é o tafetá ou a chamalote, que é furta-cor e cuja posição dos fios produz um efeito ondeado (moiré). A chamalote é, também, caracterizada como um tecido feito de pêlo ou lã de camelo, em geral com mistura de seda, conferindo o mesmo aspecto visual de moiré.

\section{0 princípio físico}

O fenômeno de moiré - também chamado de efeito, padrão ou franjas de moiré - é formado quando, segundo Oster, ${ }^{21}$ um conjunto de curvas é sobreposto a outro conjunto de curvas formando um novo conjunto. Essa sobreposição 
das grades (ou curvas) deverá ser sob um ângulo menor que $45^{\circ}$ para gerar uma interferência. A interferência formada pela interseção das linhas da grade é o que caracterizará o padrão de moiré. $5,19,21,22$
O fenômeno pode, ainda, ser gerado quando existirem pequenas diferenças na espessura e no espaçamento das malhas da grade (ou retículo), inclusive com o uso de formas circulares dos padrões de moiré (figura 1).

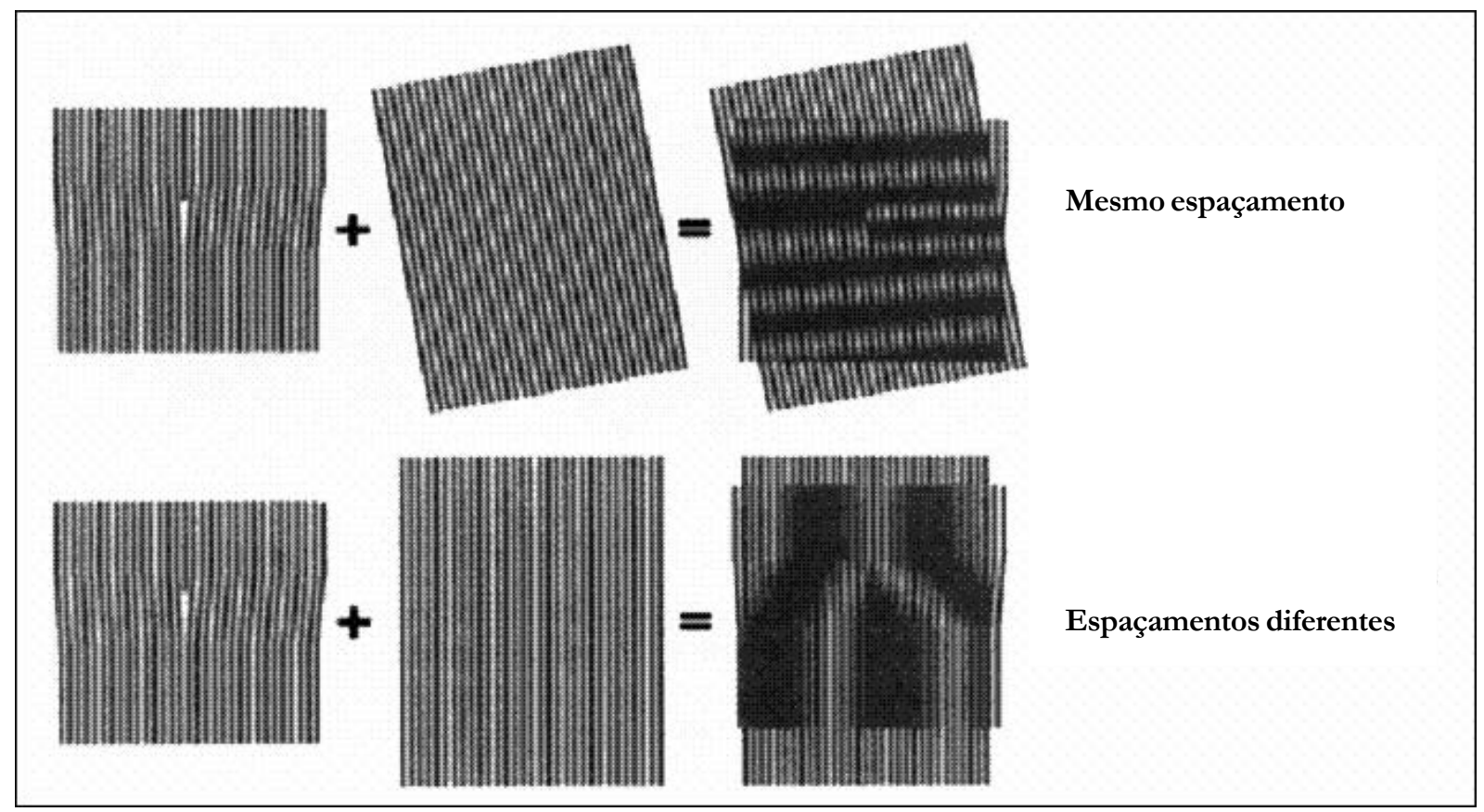

Figura 1 - Possibilidades de geração do fenômeno de Moiré, conforme período e posicionamento das grades.

Fonte: Ramirez ${ }^{23}$

Dependendo da técnica utilizada, contudo, é possível que as duas grades usadas para a geração do fenômeno de moiré não sejam objeto físico, de fato. Isso ocorre na técnica de moiré de sombra (TMS), na qual a sombra da grade projetada na superfície de análise funciona como a segunda grade $\mathrm{e}^{21,24} \mathrm{ena}$ técnica de moiré de projeção(TMP), na qual a primeira grade é projetada sobre a superfície de análise e o fenômeno óptico é gerado posteriormente por meio do processamento da imagem em soffware. ${ }^{25}$

O moiré pode ser empregado para estudar a topografia de superfícies, pois as franjas de moiré formam as linhas de contorno - ou as curvas de nível - da superfície do objeto em análise. ${ }^{2}$

\section{As técnicas de moiré}

As técnicas de moiré são um conjunto de técnicas baseadas no fenômeno físico de moiré. ${ }^{9}$
São métodos estereométricos de análise tridimensional da forma de objetos a partir de uma imagem bidimensional. ${ }^{5,7}$ Há alguns tipos de técnicas de moiré, entre elas: moiré em plano, moiré de sombra, moiré de projeção, moiré de interferometria, moiré microscópico, moiré holográfico e moiré de interferência. ${ }^{2,12}$ As técnicas diferem entre si pelo modo pelo qual o fenômeno de moiré é gerado e utilizado nas análises topográficas.

Em geral, a TMé um método simples e requer uma câmera, uma fonte de luz e uma grade. As imagens obtidas (topogramas) são formadas por franjas claras e escuras alternadas, ${ }^{5,76,27}$ sendo as escuras denominadas franjas de moiré. ${ }^{12}$ A partir dessas franjas, pode-se analisar a superfície do objeto.

Tomando como exemplo a TMS, o princípio funciona da seguinte forma: uma fonte de luz 
incide obliquamente através de uma grade na superfície de análise. Um observador (ou uma câmera fotográfica ou câmera filmadora) é posto em frente à grade. As sombras formadas na superfície de análise e vistas pelo observador (ou capturadas pela câmera) serão como um mapa topográfico, resultante de um processo óptico. ${ }^{28}$

O posicionamento da câmera fotográfica e da fonte de luz pode variar, como, por exemplo, a fonte de luz estando acima da câmera e ambas alinhadas em um mesmo plano. ${ }^{29}$ Ainda, a fonte de luz pode ser posicionada um pouco à frente $\mathrm{da}$ câmera e mais próxima à grade ${ }^{2}$. Uma alternativa é alocar a fonte de luz e a câmera fotográfica uma ao lado da outra, em um mesmo plano, porém mantendo a incidência oblíqua da luz na grade. ${ }^{6,24,30,31}$ Além disso, uma câmera fotográfica pode ser substituída por câmera filmadora. ${ }^{2,28}$

$\mathrm{Na}$ literatura, contudo, percebe-se uma carência de padronização no emprego das técnicas de moiré para a análise topográfica do corpo humano. Poucos estudos mencionam as medidas de distância entre os elementos da técnica (câmera, fonte de luz, grade, indivíduo) e outras características importantes, como detalhamento do retículo ${ }^{6,29,32}$ ou a calibração do equipamento. ${ }^{25}$ Isso se torna preocupante à medida que a replicabilidade do estudo fica comprometida por falta de informações.

Outro aspecto a ser ressaltado é que o equipamento utilizado para a TM nos diferentes estudos analisados, em sua maioria, foi de origem inespecífica, o que pode significar que foi desenvolvido pelo próprio laboratório acolhedor da pesquisa. Destacam-se alguns estudos, ${ }^{6,24,29-31}$ que utilizaram a TMS e esclareceram que as grades foram desenvolvidas pelos respectivos grupos de pesquisa, tendo também fornecido mais informações sobre elementos relativos à metodologia aplicada. Ainda assim, mesmo quando mencionado o fabricante do equipamento no estudo, como o de Mínguez et al., ${ }^{25}$ utilizando a TMP para a análise de desvios posturais da coluna vertebral em sujeitos com escoliose, não foram especificadas as características do projetor, do retículo projetado tampouco das distâncias utilizadas.

Além disso, no que tange ao esquema experimental, outras montagens do aparato utilizado na TM são permitidas, o que depende do método utilizado, dentro das várias técnicas de moiré existentes. Na TMS, por exemplo, podese optar por uma grade móvel que se movimenta no plano horizontal diante do corpo em análise $\mathrm{e}^{22}$ ou por uma grade estacionária. ${ }^{6,24,29-31}$

Quanto às características da câmera, a abertura do equipamento deve ser a suficiente para incluir, dentro do campo de visão, a grade e a sua sombra ${ }^{29}$ podendo, para tanto, se fazer uso do zoom óptico da máquina. ${ }^{6,24}$

A geração do topograma de moiré é dependente do posicionamento do objeto atrás da grade. Portanto, faz-se necessário a padronização da posição do objeto que está sendo avaliado. ${ }^{5,29}$ Este cuidado pode ser justificado, também, pela quantificação dos desvios apresentados, como a noção de profundidade, proposta por Takasaki. ${ }^{3}$

A literatura científica mostra que algumas técnicas de moiré são mais utilizadas que outras na análise da superfície do corpo humano. A TMS aparece com grande destaque na avaliação das deformidades da coluna vertebral e/ou do tronco, $, 3,4,6,24,30,31,33$, o que pode ser explicado pelo fato de, especificamente, a TMS ter sido primeiramente aplicada para a avaliação topográfica do corpo humano e, ainda, a região do tronco humano ter sido a primeira estudada através desta técnica. Porém, outras regiões corpóreas têm sido alvo de estudo com o uso da TMS, como o arco plantar, ${ }^{2,29}$ os membros inferiores $^{2} \mathrm{e}$ a região escapular. ${ }^{34}$

Por outro lado, nos últimos anos, a TMP tem surgido com mais frequência na literatura científica, principalmente nas avaliações posturais do tronco. ${ }^{7,8,25}$ Isso pode ser explicado pela atual evolução tecnológica, principalmente na área de processamento de imagens, o que permite também que a TM possa ser utilizada em análises 
topográficas de regiões corpóreas mais delicadas e complexas, como os ossos, através da Técnica de moiré de Interferometria. ${ }^{35}$

\section{Calibração do equipamento}

O procedimento de calibração na TM significa analisar a superfície de um objeto com geometria conhecida para evitar que sejam feitas marcações diretas na superfície que está sendo avaliada. ${ }^{36}$ Além disso, é possível reconstruir a geometria do objeto conhecido a partir das franjas geradas e calcular o erro padrão do instrumento prevendo a confiabilidade do equipamento utilizado para a técnica. ${ }^{6,30}$

$\mathrm{Na}$ TMS, um procedimento de calibração foi descrito por Hertz. ${ }^{6} \mathrm{Na}$ ocasião, foi utilizado um cone branco (altura $=30 \mathrm{~mm}$ ) confeccionado em TECNIL (material tipo náilon), com marcações, tal como anéis, a cada $5 \mathrm{~mm}$ de profundidade do objeto (total de seis marcações efetuadas). O cone foi afixado em uma estrutura de madeira e posicionado detrás da grade (retículo), a uma distância de $10 \mathrm{~mm}$. A imagem foi capturada a uma resolução de 96x96pp e zoom óptico da máquina fotográfica de $2 \mathrm{x}$. Após a análise das imagens, calculou-se o erro padrão do instrumento $(\mathrm{EP}=$ 0,05136), que, segundo a autora, se mostrou bastante aceitável para uma significância de 5\%. Com o procedimento de calibração, verificou-se que a distância entre cada franja formada equivalia a $4,9 \mathrm{~mm}$.
Já na TMP, uma típica calibração foi descrita por Mínguez et al. ${ }^{25}$ Segundo os autores, duas imagens obtidas da grade projetada na superfície plana (sem o objeto de análise) são capturadas em duas posições com distância de $400 \mathrm{~mm}$ entre elas. Essas imagens são chamadas de "grade da frente" e "grade de trás". Após, o indivíduo a ser avaliado é posicionado em frente àquela superfície plana e passa a corresponder à "grade de trás" (nota-se que o espaçamento estipulado em $400 \mathrm{~mm}$ é o suficiente para caber uma pessoa entre as duas grades projetadas, a "grade da frente" e a "grade de trás”). A ideia é que, após a geração das franjas de moiré na superfície do corpo do indivíduo, a grade projetada anteriormente sirva de base para os cálculos seguintes, no processamento do topograma.

Ainda quanto à TMP, a colocação de um objeto de referência ao lado do objeto cuja superfície será estudada é outra opção para que as distâncias entre as franjas sejam calibradas. Este objeto pode ser um retângulo com medidas conhecidas $^{36}$ ou qualquer outro objeto de geometria conhecida.

\section{Análise dos topogramas}

A análise médica das imagens de moiré é baseada na simetria das franjas de ambos os lados em diferentes regiões do corpo, conforme ilustra a Figura 2. ${ }^{26}$ Portanto, uma análise de predominância qualitativa. ${ }^{4}$ 


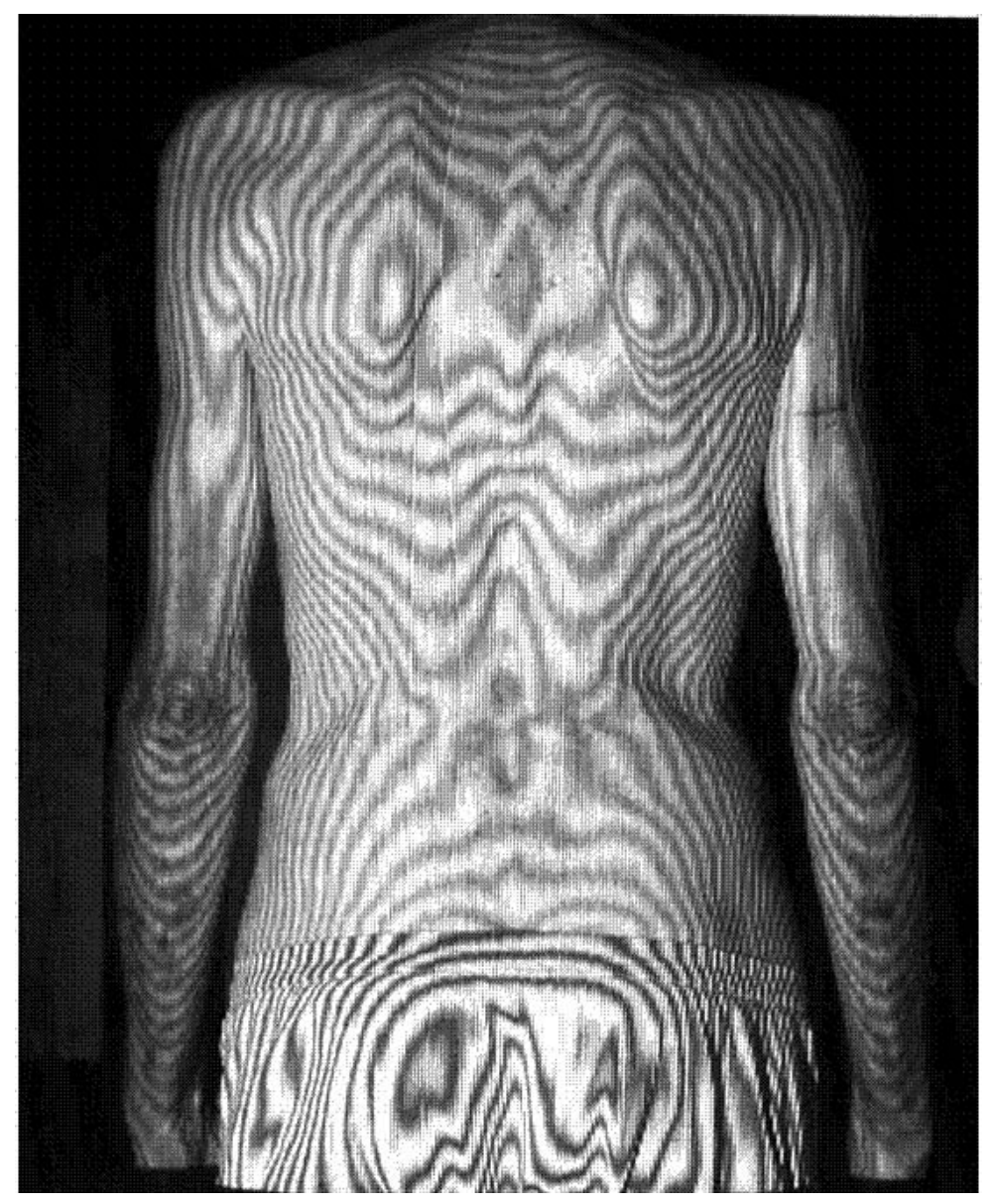

Figura 2 - Topograma de Moiré do dorso de um indivíduo idoso.

A avaliação médica qualitativa dos topogramas produzidos, baseada em inspeção visual das imagens, pode ser uma tarefa extremamente cansativa, principalmente quando se pretende avaliar deformidades posturais de um grande número de pessoas em curto período de tempo como ocorre em estudos populacionais ${ }^{24}$ ou no rastreamento de escoliose em crianças em ambiente escolar. ${ }^{4}$ Kim et al. ${ }^{7}$ e Kim et al. ${ }^{37}$ afirmaram que o cansaço provocado por uma avaliação de predominância subjetiva pode interferir no julgamento das imagens de moiré. Para tanto, segundo os autores, é necessária a automatização do procedimento de análise dos topogramas capturados do dorso de indivíduos.

Diante disso, Porto, ${ }^{24}$ em estudo populacional de avaliação postural de idosos com mais de 60 anos (Estudo Multidimensional dos Idosos de Porto Alegre, Brasil), propôs a utilização de um software livre para a análise dos topogramas dos indivíduos de modo que não detectasse automaticamente as deformidades na região dorsal da pessoa, mas que facilitasse a avaliação, fornecendo as medidas de desvio angular e profundidade com mais precisão.

A partir de fotografias com 256 tons de cinza, que variavam de 0 (preto) a 255 (branco), capturadas do dorso de cada idoso, Porto ${ }^{24}$ avaliou os desvios laterais da coluna torácica com medida dos ângulos articulares formados (em graus) e caracterização das concavidades presentes, o desalinhamento das escápulas no plano frontal com a medição dos desvios angulares, em graus, o desnivelamento das regiões escapulares através da contagem do número de centróides formados como uma noção de profundidade e, a hipercifose torácica. Para esta avaliação, a autora dispôs de software livre (Power Draw 2D Vector Application) e 
conseguiu otimizar o processo de avaliação das imagens.

Anteriormente, outros autores ${ }^{6,30,31}$ propuseram características similares de avaliação e, ainda, buscaram um parâmetro numérico nas avaliações dos sujeitos. Entretanto, nesses estudos, foi feito o uso de instrumentos mais rústicos para a análise dos desvios posturais dos indivíduos como papel, lápis ou caneta, transferidor e régua.

O aspecto limitante de uma avaliação postural com teor altamente subjetivo é que, segundo Kim et al., ${ }^{7}$ a informação numérica do grau de assimetria é útil na avaliação da deformidade da superfície corporal apresentada. Por isso, embora as tentativas de autores que tentaram quantificar os desvios posturais de maneira não automatizada ainda não sejam as melhores opções a serem seguidas, essas iniciativas mostram a preocupação do setor em melhorar esta área de avaliação biomecânica. Isso pode sugerir, inclusive, que a vontade de se aplicar uma avaliação postural baseada na TM fidedigna e vantajosa pode esbarrar em obstáculos financeiros.

A automatização do procedimento de avaliação das imagens de moiré leva vantagens como estudar um maior número de pessoas em período mais curto que se fosse realizado de modo visual, manual ou semiautomático. Kim et al., ${ }^{8}$ por exemplo, ao proporem uma nova técnica de detecção automática da escoliose, conseguiram avaliar 1.200 topogramas obtidos de indivíduos jovens. Já Porto ${ }^{24}$, avaliou 444 idosos no rastreamento de deformidades do tronco com o uso de método semi-automático de avaliação dos topogramas.

\section{Aplicações}

A TM tem as vantagens de ser um método não-invasivo e não produzir radiação como ocorre em um exame com raio-X, ${ }^{1,2}$ não necessitar de avaliadores altamente treinados para a aplicação do método por ser simples, ${ }^{1,2,26}$ permitir que muitas pessoas sejam avaliadas em curto espaço de tempo, ${ }^{1,2}$ ser replicável, ${ }^{1,26}$ apresentar baixo custo, ${ }^{2,26}$ poder substituir a avaliação com o raio-
$\mathrm{X}$ ou servir de método complementar no diagnóstico de doenças. ${ }^{2,6,31}$

A TM mostra-se bastante eficaz na análise topográfica de objetos com superfícies irregulares e não tão rígidas, como o corpo humano. ${ }^{14} \mathrm{~A}$ utilização da TM na análise topográfica do corpo humano é possível por poder identificar a localização das estruturas esqueléticas sob a pele indiretamente através do relevo. ${ }^{34}$ Devido a isso, a TM possui muitas aplicações para os profissionais da saúde nas suas avaliações clínicas de pacientes ou alunos. Essas aplicações incluem: a detecção precoce da escoliose; ;,14,38 a reconstrução não-invasiva da coluna vertebral com base nos topogramas gerados; ${ }^{22}$ a detecção de outras deformidades da coluna vertebral (escolioses, hipercifoses, hiperlordoses, dorso plano, gibosidades) em diferentes planos ortogonais (sagital, frontal e transverso) a partir de uma única imagem obtida do dorso de um indivíduo; $6,24,26,30,31$ a avaliação do arco plantar;,2,29 a caracterização da morfologia palatal e os fatores que a influenciam; ${ }^{32}$ a caracterização da forma e a detecção de anormalidades de membros inferiores; ${ }^{2}$ a descrição da forma das escápulas e a simetria com relação à coluna vertebral e entre $\mathbf{s i} ;{ }^{34}$ a verificação da influência da prática de esportes na morfologia do tronco. ${ }^{33}$

O Japão foi o pioneiro no uso da TM para o rastreamento de escolioses em crianças nas escolas. Atualmente, 11 prefeituras (de um total de 47, incluindo Tóquio) empregam a TMS para o rastreamento de escoliose nas crianças escolares. Segundo Kim et al., em 1977, o Ministro da Educação do Japão iniciou um programa de detecção precoce da escoliose em crianças e jovens nas escolas japonesas. Porém, a detecção da escoliose em estágios iniciais era feita a partir de exame clínico com a realização de flexão de tronco do paciente. Os autores consideram este teste nãoreprodutivo e não-objetivo. Portanto, a TMS se mostrou um método alternativo, através da visualização da assimetria das franjas de moiré no dorso do indivíduo. E é justamente por este motivo - a fácil visualização das franjas de moiré na pele do sujeito - que tornou o método tão popular. 
Contudo, é essencial o estabelecimento de rigor metodológico durante a aplicação do método e a análise dos topogramas sem o qual os resultados encontrados nas análises não são representativos da realidade clínica do indivíduo. Nesse sentido, a literatura aponta para estudos que objetivaram verificar a fidedignidade do método empregandoo em grande escala e comparando-se os resultados com outros métodos mais usuais ou o padrão-ouro para deformidades da coluna.

Quando se busca um método alternativo de avaliação médica, priorizando custos mais reduzidos, é necessário que o método substitutivo seja preciso e acurado. Assim, ao propor a TM como método de avaliação da superfície do corpo humano, a precisão da TM exige que haja reprodutibilidade nas medições efetuadas repetidas vezes. Estatisticamente, a acurácia de um instrumento é mais bem avaliada quando comparada a um método padrão-ouro ou a uma técnica de referência tida como acurada. ${ }^{39}$

Há uma grande carência na literatura científica de estudos que objetivaram avaliar a acurácia da TM para avaliações da superfície do corpo humano. Daruwalla \& Balasubramaniam ${ }^{40}$ avaliaram a acurácia da TM aplicada no dorso de indivíduos jovens comparando os resultados com a radiografia de cada pessoa examinada. Foram estudados 1.342 topogramas de crianças de uma escola de Singapura, obtidos em 1982. Os resultados do estudo demonstraram que a acurácia do lado das curvas foi identificada em $68 \%$ na coluna torácica, $54 \%$ na coluna tóraco-lombar e 15\% na região lombar. Os autores acreditam que o teste de flexão do tronco oferece uma ferramenta econômica mais oportuna para um programa em massa de mapeamento dos desvios posturais e a TM como um método complementar de diagnóstico.

No rastreamento de escoliose, alguns pesquisadores aplicaram a TMS em grande escala especialmente para a detecção precoce de desvios posturais em crianças e jovens em idade escolar, como Adler, Csongradi \& Bleck, ${ }^{4}$ e Yeras, Peña \& Junco. ${ }^{2}$

Adler, Csongradi \& Bleck $^{4}$ avaliaram 327 meninas, quanto à escoliose, em cinco das oito escolas públicas de Santa Clara, na Califórnia, Estados Unidos. Os autores aplicaram a TMS e estudaram a assimetria das franjas e o exame postural clínico. Comparando os dois métodos, os autores encontraram uma baixa correlação $(\mathrm{r}=0,16)$, o que foi atribuído ao sistema de moiré empregado e à não-replicabilidade da TMS em todas as escolas avaliadas.

Já Yeras, Peña \& Junco² avaliaram 203 jovens entre 10 e 15 anos de uma escola em Havana, Cuba. Os autores compararam a TMS em observação direta das franjas de moiré nas costas das crianças e em observação direta das mesmas franjas de moiré gravadas em uma fita de vídeos. Além disso, compararam a TMS com o clássico exame clínico realizado por médicos ortopedistas que incluía: exame físico completo, medida de alinhamento vertical com o uso de um fio de prumo e flexão de tronco para a detecção de escoliose idiopática. Os resultados demonstraram que a TMS se mostrou mais acurada que a tradicional avaliação clínica visual e se mostrou bastante sensível em detectar os falsos-positivos.

No que se refere ao perfil de sujeitos avaliados com a TM, é evidente o grande número de estudos envolvendo escolares como objetos de avaliação em detrimento de sujeitos de outras faixas etárias. O emprego da TM em grande escala já foi aplicado para o rastreamento de deformidades de tronco e coluna em indivíduos idosos, mas o número de estudos que focam este determinado perfil de sujeitos é ínfimo, se comparado àqueles que estudaram pessoas mais jovens. $O$ destaque vai para o Laboratório de Biomecânica Aeroespacial (NUBA, do Centro de Microgravidade, Faculdade de Engenharia, PUC-RS, Brasil) que, junto com o Instituto de Geriatria e Gerontologia e a Faculdade de Educação Física e Ciências do Desporto da PUC-RS, avaliou idosos residentes em município do Rio Grande do Sul, no Estudo Multidimensional dos Idosos de Porto Alegre. ${ }^{24}$

\section{Cuidados}

Para que haja replicabilidade das medidas, fazse necessário uma padronização do protocolo de avaliação estipulado, bem como da montagem do 
esquema experimental, ideia que é corroborada por Ikeda \& Terada. ${ }^{1}$ Segundo Drerup, ${ }^{5}$ o fato de o topograma de moiré depender da posição do objeto posto em análise atrás da grade talvez seja um agravante principalmente se for avaliada uma pessoa com deformidades importantes no tronco.

$\mathrm{Na}$ literatura científica, verificou-se que há muito mais trabalhos que tratam da aplicação clínica da TM que estudos que enfocam a acurácia do método e padronização das medidas. Uma das desvantagens é que a TM pode ser demasiadamente trabalhosa na fase de avaliação das imagens. Outros autores concordam com o fato de se haver necessidade de um sistema de análise automático das imagens de moiré. . $^{17,8,26}$

Embora, nos últimos anos, tenha se percebido maior utilização da TM em estudos de avaliação postural, muitos aspectos metodológicos não são amplamente descritos, o que compromete a

\section{REFERÊNCIAS}

1. Ikeda T, Terada H. Development of Moiré method with special reference to its application to biostereometrics. Opt Laser Technol 1981:13(6):302-6.

2. Yeras A, Peña R, Junco R. Moiré topography: alternative technique in health care. Opt Lasers Eng. 2003;40(1-2):105-16

3. Takasaki H. Moiré Topography. Appl Opt. 1970; 9(6):1467-72.

4. Adler N, Csongradi J, Bleck E. School screening for scoliosis : one experience in California using clinical examination and Moiré Photography. West J Med. 1984; 141(5):631-3.

5. Drerup B. A procedure for the numerical analysis of Moiré topograms. Photogramm 1981;36(2):41-9.

6. Hertz H. Construção a calibração da técnica de Moiré de sombra para análise postural. Porto Alegre. monografia [ Pós Graduação em Biomédica]— Pontifícia Universidade Católica do Rio Grande do Sul; 2005.

7. Kim H, et al. Automatic scoliosis detection based on local centroids evaluation on Moiré topographic images of human backs. IEEE Trans Med Imaging 2001; 20(12):1314-20. replicabilidade do método. Outra aspecto limitante deste estudo foi que parece não haver uma padronização nos procedimentos de teste.

\section{CONCLUSÕES}

Este estudo tratou de revisar os aspectos referentes à TM, os quais foram embasados em obras científicas publicadas a partir de 1970, quando a técnica passou a ser utilizada com propósitos médicos. O método mostra-se bastante versátil em suas aplicações clínicas e extremamente útil como complemento nos diagnósticos médicos, apresentando vantagens no emprego da técnica (facilidade), nos custos envolvidos, no tempo de coleta e na análise das imagens. Apesar disso, percebeu-se uma lacuna encontrada na literatura pesquisada relacionada à carência de estudos que avaliaram a topografia do corpo de pessoas idosas e de estudos que enfocam a acurácia da TM.

8. Kim H, et al. Scoliosis detection based on difference of apexes position and angle on Moiré topographic images. Int Congr Ser 2004; 1268:94.

9. Lino A, Fabbro I. Determinação da topografia de uma fruta pela técnica de Moiré de sombra com multiplicação de franjas. Ciênc Agrotec. 2004; 28(1):119-25.

10. Porto F, et al. Postural evaluation of men aged between 60 and 65 years old of Porto AlegreRS based on Shadow Moiré Technique. Ouro Preto : UFMG; 2007.p.566-9.

11. Porto F, Gurgel J, Sepúlveda G, Souza A,. Topografia de moiré para a avaliação postural de homens idosos de Porto AlegreRS. Mostra de Pesquisa da Pós-Graduação da PUCRS. Porto Alegre,: EAD-PUCRS; 2007.

12. Rössler T, Hrabovský M, Pochmon M. Moiré methods for measurement for displacement and topography. Czech J Phys 2006; 56(2):101216.

13. Takasaki H. Simultaneous all-around measurement of a living body by Moiré topography. new York :Helsinki ;1976. p.152734. 
14. Takasaki H. Moiré topography from its birth to practical application. Opt Lasers Eng 1982; $3(1): 3-14$.

15. Takasaki H. Moire Topography. Appl Opt. 1973; 12(4):845-50.

16. Kleiman A. Leitura: ensino e pesquisa. Campinas: Pontes ;1989.

17. Bardin L. Análise de conteúdo. Lisboa,: Edições $70 ; 2000$.

18. Porto F. Produção do Journal of Biomechanics entre os anos de 2000 e 2001 relacionada ao tema equilíbrio corporal. Rio de Janeiro: Universidade do Estado do Rio de Janeiro; 2003.

19. Bartl J, Fíra R, Hain M. Inspection of surface by the Moiré method. Meas Sci Rev. 2001;1(1):29-32.

20. Sciammarella C. The Moiré method : a review. Exp Mech. 1982:22(1)418-33.

21. Oster G. Moiré pattern. I Optics source book : Science reference series. Usa :McGraw-Hill; 1988 p. 379-81.

22. Dzielinski A, Skoneczny S, Zbikowski,. Cellular neural network application to Moiré pattern filtering. Budapest : IEEE;1990. p .139-48.

23. Ramirez A. Mini-curso: caracterização e fabricação de nanoestruturas. 2006 [Acesso em 30 ago 2007 ]. Disponível em: http:// www.cbpf.br/ labmag/MinicursoNano/ Ramirez2_CBPF2006.pdf

24. Porto F. Avaliação postural dos idosos de Porto Alegre-RS com o uso da Técnica de Moiré de Sombra. Rio Grande do Sul.Tese[ Doutorado em Gerentologia Biomédica] - PUCRS; 2008.

25. Mínguez M, et al. Quantifier variables of the back surface deformity obtained with a noninvasive structured light method: evaluation of their usefulness in idiopathic scoliosis diagnosis. Eur Spine J. 2007;16(1):73-82.

26. Batouche M, Benlamri R. A computer vision system for diagnosing scoliosis. . Orlando,: IEEE; 1994. p.2623-28.

27. Batouche M, Benlamri R, Kholladi M. A computer vision system for diagnosing scoliosis using Moiré images. Comput Biol Med. 1996; 26(4):339-53.

28. Turner-Smith A. Structured light surface measurement techniques. In: Orr J, Shelton J. Optical measurement methods in biomechanics. Germany: Chapman \& Hall; 1997.p.39-58.

29. Hamra A, Volpon J. Fotopodometria "quantitativa” na avaliação do arco plantar longitudinal medial. Rev Bras Ortop 1995; 30(8):609-14.

30. Hertz H, et al. Construção de um protótipo para análise postural através da técnica de moiré de sombra. João Pessoa : CBB; 2005.

31. Hertz H, et al.Desenvolvimento da Técnica de Moiré de Sombra como alternativa de baixo custo para análise postural. Scientia Medica. 2005;15(4):235-42.

32. Kilpeäinen P, Laine-Alava M, Lammi S. Palatal morphology and type clefting. Cleft Palate Craniofac J. 1996; 33(6):477-82.

33. Uetake U, Ohtsuki F, Tanaka H. The vertebral curvature of sportsmen. J Sports Sci. 1998;16(7):621-8.

34. Chalupová M. Use of a biomechanical model of the scapula region for the identification of muscle disbalance. 2001 [Acesso em 30 jul 2005 ]. Disponível em: mms.tudelft.nl/dsg/intersg/ Proceedings_2001_files/06_chalupova.pdf

35. Wood J, Saha S, Rao R. Investigation of nonhomogenous strain distributions in bone using Moiré Interferometry. 2001 [Acesso em 30 jul 2005 ]; Disponível em: http://www.cgl.ucsf.edu/ Research/genentech/canpredict/ references.html

36. Ortiz M, Patterson E. Location and shape measurement using a portable fringe projection system. Exp Mech. 2005;45(3):197-204.

37. $\mathrm{Kim} \mathrm{H}$, et al. Automatic judgment of spinal deformity based on back propagation on neural network. IJICIC. 2006; 2(6):1271-9.

38. Pearsall D, Reid J, Hedden D. Comparison of three noninvasive methods for measuring scoliosis. Phys Ther 1992; 72(9):648-57.

39. Hulley S, Newman T, Cummings S. Escolhendo os sujeitos de estudo: especificação, amostragem e recrutamento. In: Hulley S,,et al. Delineando a pesquisa clínica. Porto Alegre: Artmed; 2003. p.55-68.

40. Daruwalla J, Balasubramaniam P. Moiré topography in scoliosis: its accuracy in detecting the site and size of the curve. J Bone Joint Surg. 1985;67(2):211-3. 